INGENIOUS 



\title{
INGENIOUS
}

\section{The Unintended Consequences of Human Innovation}

PETER GLUCKMAN • MARK HANSON

\author{
III \\ III \\ Harvard University Press \\ Cambridge, Massachusetts \\ London, England


Copyright (C) 2019 by Peter Gluckman and Mark Hanson ALL RIGHTS RESERVED

Printed in the United States of America

First printing

Design by Tim Jones

Photograph (C) Jonathan Knowles / Stone / Getty Images

9780674242456 (EPUB)

$9780674242463(\mathrm{MOBI})$

9780674242449 (PDF)

The Library of Congress has cataloged the printed edition as follows:

Names: Gluckman, Peter D., author. | Hanson, Mark A., author.

Title: Ingenious : the unintended consequences of human innovation / Peter Gluckman and Mark Hanson.

Description: Cambridge, Massachusetts : Harvard University Press, 20I9. |

Includes bibliographical references and index.

Identifiers: LCCN 20I90I4I47 | ISBN 9780674976887 (cloth)

Subjects: LCSH: Human evolution. | Technological innovations-Health aspects. |

Civilization, Modern-Health aspects.

Classification: LCC GN28I.4 .G58 20I9 | DDC 599.93/8-dc23

LC record available at https://lccn.loc.gov/20I90I4I47 